\title{
Sistematização da assistência de enfermagem: vantagens e dificuldades na sua aplicação sob a ótica de enfermeiros
}

\author{
Systematization of nursing assistance: advantages and difficulties in its aplication under the optics \\ of nurses
}

Sistamatización de la assistencia de enfermería: ventajas y dificultades en su aolicación bajo la óptica de enfermeros

Priscila Martins e Andrade ${ }^{1,2}$ *, Elyrose Sousa Brito Rocha ${ }^{2}$, Sabrina Maria Ribeiro Amorim², Aziz Moisés Alves da Costa ${ }^{2}$, Teresa Amélia Carvalho de Oliveira ${ }^{2}$, Lorena Lívia Nolêto ${ }^{2}$, Taynnar Barbosa Ribeiro², Antonia Adrielly Sousa Nogueira ${ }^{2}$, Vitor Kauê de Melo Alves ${ }^{2}$, Daniel de Macêdo Rocha ${ }^{2}$.

\section{RESUMO}

Objetivos: Analisar a percepção do enfermeiro a respeito da Sistematização da Assistência de Enfermagem (SAE) no hospital em que trabalha, verificar se a SAE interfere na qualidade da assistência e identificar as principais dificuldades do enfermeiro para realiza-la. Metodologia: Estudo qualitativo, do tipo descritivo. Os dados foram coletados entre os meses de maio e junho de 2017, mediante entrevista com 30 enfermeiros de um hospital público de Teresina. Resultados: Houve predomínio de profissionais do sexo feminino 27 (90\%), com idade entre 25 e 60 anos e tempo de serviço entre 1 e 27 anos. Além disso, 22 (73,33\%) enfermeiros tiveram contato com a Sistematização da Assistência de Enfermagem durante a graduação. Os discursos dos enfermeiros permitiram a construção de três categorias temáticas: "A sistematização da assistência de enfermagem vista como processo que permite, aos profissionais, organizar e individualizar o cuidado"; "Percepção dos enfermeiros acerca das contribuições da sistematização da assistência de enfermagem para a melhoria do cuidado" e "Dificuldades vivenciadas por enfermeiros no processo de sistematização da assistência". Conclusão: Constatou-se que a sistematização é um instrumento que possibilita o planejamento de cuidados individualizados, organiza o serviço e proporciona autonomia profissional. Todos os entrevistados acreditam que as ações sistematizadas contribuem para melhoria do serviço, ao passo que direciona o cuidado ao paciente e aproxima os profissionais de saúde, como equipe, bem como aumenta o vínculo profissional/paciente. Como barreiras e desafios, foram citados a sobrecarga de trabalho, o despreparo da equipe e a informatização desse instrumento.

Palavras-chave: Assistência de enfermagem, Teorias de Enfermagem, Processo de Enfermagem.

\begin{abstract}
Objectives: To analyze the nurses' perception about the Systematization of Nursing Care (SNC) in the hospital in which they work, to verify if the SNC interferes in the quality of the care, to make the nurse's point of view and to identify as the main difficulties of the nurse to perform an SNC. Methodology: Qualitative study, of the descriptive type. The data were collected in the months of May and June of 2017, by interview with 30 nurses of a public hospital in Teresina. Results: The study showed that the professionals interviewed are 27 (90\%) female, aged between 25 and 60 years and service time between 1 and 27 years. In addition, 22 (73.33\%) of the nurses had contact with SNC during graduation. Nurses' discourses allow the construction of three thematic categories: "The systematization of nursing care as a systematic process that allows professionals to organize and individualize the care provided"; "Nurses' perception about the contributions of the systematization of
\end{abstract}

${ }^{1}$ Associação Piauiense de Combate ao Câncer. *E-mail: pmandrade02@hotmail.com

2 Universidade Estadual do Piauí (UESPI), Teresina - PI. 
nursing care for care" and "Difficulties experienced by nurses in the process of systematization of care". Conclusion: It was verified that for nurses systematization is an instrument that allows the planning of individualized care, organization and autonomous service to the professional. All the interviewees believe that the SNC contributes to improve the service, while it directs care to the patient, gives support and autonomy to the nurse and brings health professionals together as a team, as well as increasing the professional / patient relationship. Barriers were cited for a systematization, overwork, unprepared staff and computerization of SNC.

Keywords: Nursing Care, Nursing Theory, Nursing Process.

\section{RESUMEN}

Objetivos: Analizar la percepción del enfermero sobre la SAE en el hospital en que trabaja, verificar si la Sistematización de la Asistencia de Enfermería (SAE) interfiere en la calidad de la asistencia e identificar las principales dificultades del enfermero para realizarla. Metodología: Estudio cualitativo, del tipo descriptivo. Los datos fueron recolectados en los meses de mayo y junio de 2017, mediante entrevista con 30 enfermeros de un hospital público de Teresina. Resultados: El estudio evidenció que los profesionales entrevistados son $27(90 \%)$ del sexo femenino, edad entre 25 y 60 años y tiempo de servicio entre 1 y 27 años. Además, 22 $(73,33 \%)$ de los enfermeros tuvieron contacto con la SAE durante la graduación. Los discursos de los enfermeros permitieron la construcción de tres categorías temáticas: "La sistematización de la asistencia de enfermería vista como proceso sistemático que permite, a los profesionales, organizar e individualizar el cuidado prestado"; "Percepción de los enfermeros acerca de las contribuciones de la sistematización de la asistencia de enfermería para la mejora del cuidado" y "Dificultades vivenciadas por enfermeros en el proceso de sistematización de la asistencia". Conclusión: Se constató que para los enfermeros la sistematización es un instrumento que posibilita la planificación de cuidados individualizados, organiza el servicio y proporciona autonomía al profesional. Todos los entrevistados creen que la SAE contribuye a la mejora del servicio, mientras que dirige el cuidado al paciente, da respaldo y autonomía al enfermero y aproxima a los profesionales de la salud, como equipo, así como aumenta el vínculo profesional / paciente. Como barreras, fueron citadas: la sobrecarga de trabajo, el despreparo del equipo y la informatización de la SAE.

Palabras clave: Atención de Enfermería, Teoría de Enfermería, Proceso de Enfermería.

\section{INTRODUÇÃO}

Entende-se por Enfermagem a arte de cuidar do indivíduo, da família e da comunidade de forma integral e holística. No decorrer dos anos, a área da Enfermagem acumulou novos conhecimentos e teorias assistenciais foram formuladas. Surge então a Sistematização da Assistência de Enfermagem (SAE), criada com o objetivo de organizar e sistematizar os cuidados apoiando-se em conhecimentos científicos, dando ao enfermeiro base para identificar as necessidades de cada cliente, além de promover maior segurança e qualidade à assistência prestada (CHAVES RG, et al., 2016).

No Brasil, os profissionais de Enfermagem ambicionaram sistematizar a sua assistência a partir da década de 70. Esses se basearam nas ideias da teórica Wanda Horta, que propôs o Processo de Enfermagem (PE), instrumento de cunho científico utilizado pela equipe de enfermagem para oferecer o cuidado sistematizado. O PE consiste em cinco etapas: coleta de dados, diagnóstico de enfermagem, planejamento de enfermagem, implementação e avaliação (SANTOS JÁ, et al., 2015).

De acordo com a Resolução do Conselho Federal de Enfermagem (COFEN) 358 de 2009, que dispõe sobre a SAE e a implementação do Processo de Enfermagem (PE), este deve ser realizado, de forma deliberada e sistemática, em todos os ambientes, públicos ou privados, em que ocorre o cuidado profissional de Enfermagem. Ao enfermeiro, cabe a liderança na execução e avaliação do PE, de forma que alcance os resultados esperados, é privativo à ele o diagnóstico de Enfermagem acerca das respostas do indivíduo, família ou coletividade em um dado momento do processo saúde-doença, assim como a prescrição das ações ou intervenções de Enfermagem a serem realizadas. 
De acordo com Soares Ml et al. (2015), a SAE é o instrumento utilizado pelo enfermeiro em sua assistência, o qual colabora assegurando a qualidade do serviço realizado pela equipe de enfermagem, fundamentando o mesmo em teorias científicas para que desta forma sejam alcançados resultados positivos para a saúde dos pacientes. Sendo assim, acredita-se ser imprescindível que o enfermeiro conheça seu instrumento de trabalho para que possa aplica-lo da melhor forma possível.

Porém, mesmo com os benefícios que a SAE traz, algumas barreiras devem ser ultrapassadas para que sua implantação possa ser de todo satisfatória. Parte dos enfermeiros consideram a SAE um instrumento complexo, é identificado também um despreparo da equipe de Enfermagem. Outra dificuldade apontada por enfermeiros é a sobrecarga de trabalho, o dimensionamento inadequado de funcionários em relação ao fluxo de pacientes (MARIA MA, et al., 2012).

Diante do exposto, o presente trabalho teve como objetivo analisar a percepção do enfermeiro a respeito da SAE no hospital em que ele trabalha, verificando se a SAE interfere na qualidade da assistência, do ponto de vista do enfermeiro e Identificando as principais dificuldades do enfermeiro para realiza-la.

\section{METODOLOGIA}

Trata-se de um estudo de abordagem qualitativa, do tipo descritivo, realizado em um hospital de referência do município de Teresina-PI. Participaram deste estudo 30 enfermeiros distribuídos entre todos os setores e turnos de trabalho.

Foram utilizados como critérios de inclusão: ser enfermeiro, de ambos os sexos, aceitar participar da pesquisa e ter tempo igual ou superior a seis meses de serviço prestado à instituição, não foram incluídos os profissionais que estavam afastados no período da coleta de informações (licenças, férias ou outros motivos), e profissionais voluntários.

A coleta das informações ocorreu no período de maio e junho de 2017, por meio da aplicação de uma entrevista aberta, com roteiro elaborado pelos autores, realizada e gravada no Posto de Enfermagem ou em sala reservada, dependendo da estrutura física do setor.

Todos os profissionais que se enquadraram nos critérios de inclusão foram entrevistados, até que houvesse a saturação das informações, identificada pela repetição das mesmas. As entrevistas somente ocorreram após a assinatura do Termo de Consentimento Livre e Esclarecido-TCLE.

Os discursos coletados foram transcritos, organizados e analisados, utilizando-se a técnica de análise de conteúdo, que constitui-se em um conjunto de instrumentos metodológicos que se aplica a discursos diversificados. Em seguida, categorizados segundo a investigação dos temas que surgiram e a análise temática, por serem eficazes na investigação de discursos diretos (BARDIN L, 2009).

O estudo atendeu aos aspectos éticos e legais do Conselho Nacional de Saúde, a pesquisa foi submetida ao Comitê de Ética em Pesquisa (CEP) da Universidade Estadual do Piauí (número do parecer: 1.900.498) através do cadastro na Plataforma Brasil, com aprovação da instituição co-participante e seguiu todas as diretrizes da Resolução de № 466 de dezembro de 2012.

\section{RESULTADOS E DISCUSSÃO}

Entre os enfermeiros que participaram do estudo, o sexo predominante foi o feminino, com 27 (90\%). A faixa etária desses profissionais situou-se entre 25 e 60 anos e o tempo de serviço na instituição variou entre 01 e 27 anos. Em relação ao contato com SAE na graduação $22(73,33 \%)$ enfermeiros, afirmaram ter vivenciado essa experiência. No entanto, 8 (26,66\%) enfermeiros, formados há mais de 10 anos, não tiveram, já que a SAE ainda não havia sido implantada nas instituições.

A partir dos questionamentos realizados, os discursos dos enfermeiros permitiram a construção de três categorias temáticas: "A sistematização da assistência de enfermagem vista como processo que permite aos 
profissionais organizar e individualizar o cuidado"; "Percepção dos enfermeiros acerca das contribuições da sistematização da assistência de enfermagem para a melhoria do cuidado" e "Dificuldades vivenciadas por enfermeiros no processo de sistematização da assistência".

\section{A sistematização da assistência de enfermagem vista como processo que permite aos profissionais organizar e individualizar o cuidado.}

Dentre os enfermeiros que participaram do estudo, nove (30\%) afirmaram que a SAE são os cuidados a serem prestados ao paciente de acordo com sua necessidade, em um dado momento em que o mesmo se encontra, tornando possível o planejamento de cuidados individualizados como pode-se perceber nas falas a seguir:

"A SAE é a prestação de cuidados adequados para cada paciente naquele momento que ele se encontra, a indicação do cuidado adequado de acordo com a situação que ele se encontra naquele momento." (E13)

"A sistematização da assistência de enfermagem é uma forma de priorizar o atendimento com o cliente, então ela é uma forma individualizada de você ver cada cliente" (E25)

Em acordo com os relatos acima, que demonstram percepção da SAE voltada ao cuidado individualizado, Silva CG et al. (2016) afirmam que a SAE é uma estrutura de organização que tem o objetivo de dinamizar o trabalho da equipe de enfermagem, ela direciona as práticas de cuidar de maneira planejada e individualizada, buscando atender as particularidades de cada cliente, seja ele pessoa, família ou coletividade.

Sena AC et al. (2017) faz referência à teoria das Necessidades Humanas Básicas, de Wanda de Aguiar Horta, que afirma que as necessidades do ser humano estão intimamente ligadas, não podendo ser divididas, de forma que se uma delas se manifestar, todas as outras sofrem alteração. Sendo assim, as prioridades para a assistência de enfermagem devem estar em processo contínuo de avaliação e ajuste, conforme as necessidades do cliente.

Outros seis (20\%) participantes do estudo, afirmaram que a SAE é um processo sistemático, através do qual possibilita a organização e padronização do serviço da equipe de enfermagem:

"É de uma certa forma é a organização do serviço." (E4)

"A SAE pra mim é aquele modelo de organização, de todo o passo a passo do que se deve fazer com o paciente, direcionando as ações." (E10)

"O que eu entendo é que é um processo sistemático que deve ser feito né, levantamento do histórico, fazer o diagnóstico, o planejamento, as intervenções e avaliar.” (E1)

Por outro lado, dois participantes (6,66\%) ligaram o conceito de SAE especificamente às ações de cuidados prescritos, quando afirmaram que a SAE é um instrumento utilizado com o objetivo de guiar os técnicos de enfermagem, direcionando os mesmos para os procedimentos a serem realizados com determinado paciente:

"Eu vejo como um mecanismo pra nos ajudar a auxiliar melhor o técnico nos procedimentos com o paciente né, é a condução da assistência à esse paciente" (E14)

Como foi visto no relato E14, parte dos enfermeiros acredita que a SAE seja um instrumento utilizado para guiar o trabalho do técnico de enfermagem. Apesar de se saber que o objetivo da SAE seja mais amplo, reconhece-se que este profissional é parte atuante no processo de sistematização, como mostra Salvador et al. (2015), quando afirmam que o técnico é componente fundamental na equipe de enfermagem, visto que o mesmo auxilia o enfermeiro em suas ações e planejamento, ressaltando que as atividades exercidas pelo técnico de enfermagem são descritas na Lei do Exercício Profissional, e estas são: assistir o enfermeiro no planejamento, programação, orientação e supervisão das atividades de assistência de enfermagem. 
Embora perceba-se que o enfermeiro conhece todo o Processo de Enfermagem e seus passos operacionais, o discurso E14 demonstra percepção fragmentada por parte do profissional, a respeito da SAE em sua prática diária. Cabe lembrar que, para desenvolver a SAE, tem-se o processo de enfermagem (PE), que é amplo e objetiva principalmente assistência individualizada, de qualidade e direcionada às necessidades do paciente, implementado pela Resolução do Cofen 358 de 2009. Este deve ser realizado de forma deliberada e sistemática, em todos os ambientes, públicos ou privados, em que ocorre o cuidado do profissional de enfermagem (MARINELLI NP, et al., 2015).

\section{Percepção dos enfermeiros acerca das contribuições da sistematização da assistência de enfermagem para a melhoria do cuidado.}

Todos os enfermeiros entrevistados afirmaram que a SAE contribui para a melhoria da assistência da equipe de enfermagem, ao passo que direciona e permite a continuidade do cuidado, como também organiza o serviço. E esses foram alguns dos benefícios citados, pelos profissionais, sobre a SAE.

Desta forma, quando questionados de que forma a sistematização da assistência contribui para melhorar o serviço, 18 (60\%) enfermeiros declararam que, a SAE direciona o cuidado ao paciente, de acordo com as necessidades do mesmo, tal como facilita a visualização dos resultados alcançados a partir do plano de cuidados realizado para determinado paciente, como se vê nos relatos a seguir:

"Contribui porque nosso trabalho ele é pautado na ciência, não é um trabalho à esmo, então a gente consegue efetuar ações planejadas junto com a nossa equipe à qual a gente direciona para dar o que o paciente precisa." (E11)

"Me facilita a ver a evolução desse paciente, e isso me abre portas pra ver que meios eu uso pra acelerar na recuperação desse paciente" (E14)

"Porque quando você planeja uma coisa, fica bem mais fácil você visualizar os resultados, bem melhor, se eu não planejo se eu não tenho um norte do que eu estou fazendo, ai eu também não vou poder ver esses resultados, é o que a gente vê quando você planeja a assistência." (E29)

Ainda sobre as contribuições da SAE para a melhoria do cuidado, 4 (13,33\%) enfermeiros relataram que a sistematização favorece a assistência, pois é uma forma de respaldo para o profissional de enfermagem à medida que este registra todo e qualquer procedimento realizado. Bem como imprime autonomia à classe de enfermeiros, pois dá maior visibilidade para a profissão:

"De certa forma ela traz mais autonomia para o enfermeiro, porque a gente sabe que dentro do ambiente hospitalar o enfermeiro não tem tanta autonomia, e a partir do momento que a SAE passou a ser cobrada, a gente tem a obrigação de avaliar o paciente para definir diagnóstico, a gente pode dar um norte diferente para o tratamento do paciente." (E9)

"Porque está tudo registrado, o que a faz e deixa de fazer com o paciente, fica tudo protocolado." (E15)

Dentre os 30 participantes deste estudo, 7 (23,33\%) referiram também que a SAE melhora a comunicação entre a equipe, conforme são feitas as anotações no prontuário do paciente. Mencionaram ainda que a sistematização aproxima não só os integrantes da equipe em si, mas também aproxima o profissional do cliente:

"Melhora, porque se a gente quiser anotar alguma coisa, a gente tem que ir lá ver o paciente, examinar o paciente, então de uma certa forma a gente tenta se envolver com o paciente." (E4)

"É uma continuação pro colega do outro turno, ele consegue ver o que aconteceu com o paciente durante o dia" (E15)

"Melhora, assim é uma forma pra gente ter o controle da assistência, dos procedimentos, de evolução do paciente também, e não só para os enfermeiros, para os técnicos também." (E16) 
Segundo Silva CG et al. (2013) é importante ressaltar o uso do processo de enfermagem, pois orienta o trabalho do enfermeiro na coleta de dados, a partir da qual é possível identificar as necessidades do cliente, para assim o profissional propor intervenções e, em seguida, implementá-las e avaliar os resultados alcançados.

Como já visto o processo de enfermagem se divide em cinco etapas, uma delas é o planejamento de enfermagem, por meio dele o enfermeiro organiza e direciona sua assistência, traçando seu plano de cuidados (SANTOS JA, et al., 2015). Como pode se perceber na fala E29, os profissionais entendem que com a sistematização é possível identificar um diagnóstico mais efetivo, facilitando o direcionamento do cuidado.

Por outro lado, o discurso E4 traduz ainda uma percepção incompleta e frágil da organização da assistência que a SAE é capaz de promover, uma vez que o "envolver-se com o paciente", através das ações de entrevista e exame físico por exemplo, faz parte da sequência natural do cuidado sistematizado, não se limitando apenas ao objetivo de ter que compor a anotação de enfermagem.

Acredita-se que a sistematização da assistência de enfermagem, além de beneficiar o paciente, traz melhorias para a profissão do enfermeiro, pois este se sente mais seguro em executar seu serviço, bem como mais autônomo em suas ações, como pode ser notado no relato E9.

Resultados semelhantes foram encontrados na pesquisa de Medeiros AL et al. (2013), que em seu estudo constataram que os enfermeiros entendem a SAE como uma metodologia de trabalho que organiza e direciona as ações de enfermagem, voltadas para as necessidades individuais do cliente, assim como garante autonomia, segurança e maior visibilidade ao profissional, visto que a prática da enfermagem, com a sistematização, passa a ser pautada por teorias cientificas, o que dá ao profissional confiança em seu trabalho e a certeza que tomará decisões assertivas no tratamento de seu cliente.

Os enfermeiros relataram ainda, que a SAE melhora a comunicação entre a equipe, como apontado no relato E15. A partir da sistematização é possível trocar informações sobre determinado paciente. Os profissionais acreditam que a SAE trouxe melhorias para a comunicação entre a equipe, visto que as informações acerca do paciente são difundidas, de forma eficiente, entre os membro da equipe, facilitando a continuidade do cuidado.

\section{Dificuldades vivenciadas por enfermeiros no processo de sistematização da assistência.}

Em relação às dificuldades encontradas pelos enfermeiros para realizar a SAE, $22(73,33 \%)$ dos profissionais, apontaram a pouca quantidade de profissionais, resultando em uma sobrecarga de trabalho. Mencionaram ainda a falta de tempo para realizar suas funções de forma plena. Sabe-se que em uma clínica o enfermeiro exerce tanto a assistência quanto a gerência do serviço, e um ponto citado é o fato do profissional se dividir entre o cuidado com o paciente e a burocracia que o serviço demanda, como pode-se perceber nas falas a seguir:

"Nós temos poucos profissionais pra atender muitos pacientes, então fica muito difícil né, pra você executar todos os protocolos que é necessário, a equipe fica sobrecarregada" (E2)

"O tempo, o tempo assim porque é muita coisa, muita burocracia, as vezes a gente pega essa clínica, e pega mais outra, então são mais de 30 pacientes, e é aquela correria" (E28)

"A parte burocrática termina tomando muito a gente em detrimento da assistência, a gente se bate muito entre a assistência e a burocracia pra que a gente consiga desenvolver todo o nosso processo." (E30)

A função do enfermeiro, na realidade hospitalar brasileira, se divide em assistencial e gerencial, porém como visto na fala E28, muitas vezes o enfermeiro fica responsável por muitos pacientes, dificultando a aplicação do processo de enfermagem de forma satisfatória. Segundo Tavares TS et al. (2013), para que se tenha uma assistência de enfermagem adequada e individualizada, é essencial que a implantação da SAE se 
ajuste à realidade da instituição, adequando-se ao número de funcionários, tipo e intensidade de cuidados demandado pelos pacientes ali internados.

Para obter o número adequado de funcionários em um serviço, é necessário realizar o dimensionamento de pessoal. Através dele é possível prever a quantidade de profissionais necessária para suprir as necessidades da assistência de enfermagem. Os aspectos que deve-se ter em vista para este cálculo é previsto na Resolução (COFEN) o 293 de 2004, que dispõe sobre o dimensionamento dos profissionais de enfermagem e deve levar em conta características referentes à instituição, como sua missão, estrutura física e organizacional e tipos de serviços; bem como referentes ao serviço de enfermagem, dinâmica de funcionamento das unidades nos diferentes turnos, indicadores de avaliação da qualidade da assistência e jornada de trabalho e, por fim, características referentes à clientela, podendo-se usar o sistema de classificação de pacientes (SCP) e realidade sócio-cultural e econômica.

A resistência por parte dos técnicos de enfermagem em checar a prescrição de enfermagem, foi indicada por $15(50 \%)$ dos enfermeiros, os quais acreditam que essa resistência seja mais forte dentre os técnicos que se formaram antes da sistematização despontar como ferramenta necessária para o cuidado. Os enfermeiros reportaram também a falta de preparo dos profissionais, e aqui ressaltaram que não são apenas os técnicos, mas também os próprios enfermeiros, que desconhecem o processo de enfermagem:

"É a questão ainda dos técnicos serem um pouco resistentes pra tá fazendo naqueles horários o que a gente determina na SAE né, e também os próprios enfermeiros, os próprios colegas que ainda não acham que é uma coisa importante pro paciente." (E16)

"Os técnicos tem muita resistência, eles também não se preocupam muito em checar o que a gente faz." (E8)

"O que eu vejo é que principalmente os profissionais que tem mais idade já são um pouco mais antigos na profissão, eles tem um pouco mais de resistência quanto à SAE." (E21)

Os enfermeiros ressaltam que os técnicos de enfermagem mais antigos no serviço, com maior tempo de formação, são os mais relutantes em realizar a sistematização de forma adequada, como é possível ler na fala E8 e E21. Talvez por não terem experienciado a SAE durante sua formação, não compreendem a importância dela na prática do cuidado do enfermo. Para Casafus KCU et al. (2013), os auxiliares/técnicos de enfermagem não veem a necessidade de realizar a SAE, em razão da autoafirmação de seu conhecimento para a execução dos cuidados, acreditando ser a sistematização uma atividade que não está relacionada à prática.

Além dos técnicos de enfermagem, é possível perceber no relato E16 e E9, que os próprios enfermeiros não entendem a SAE como parte importante no processo de enfermagem. Durante a coleta de dados podese notar que os enfermeiros consideram a sistematização um instrumento extenso, que toma muito o tempo do profissional:

"Ela exige muito tempo, ela faz com que a gente perca muito tempo nos papéis." (E9)

Apenas uma enfermeira $(3,33 \%)$ citou o sistema informatizado como barreira na realização da SAE, conforme sua fala:

"A barreira que eu encontro é porque a gente tá fazendo pelo sistema MV, que é informatizado, ele facilitou porque é mais prático, a dificuldade é o computador dar problema." (E17)

A informatização do sistema foi apontada somente por um enfermeiro como barreira para realizar a SAE, devido falhas no computador. Vive-se a era tecnológica, a qual já está inserida na área hospitalar, demandando dos profissionais conhecimento para utilizar essa nova ferramenta de trabalho. Ribeiro JC et al. (2014), em sua pesquisa observaram que os enfermeiros acreditam que a informatização facilitou o trabalho do enfermeiro à medida que, facilita o monitoramento geral das ações, a comunicação entre equipes de saúde 
de diversos setores de internação, a otimização do tempo gasto e a maior segurança para o registro de enfermagem.

No entanto, existem aspectos que dificultam o uso da SAE informatizada, a baixa manutenção do sistema e a dificuldade na sua atualização. Ribeiro JC et al. (2014), ressaltam ainda que à proporção que o enfermeiro "alimenta" o sistema, este processa as informações e gera diagnósticos, tomando do enfermeiro seu raciocínio clínico, a partir do qual ele elabora sua prescrição e analisa os resultados. Sendo assim é necessário o treinamento profissional para o uso da sistematização da assistência informatizada, além dos recursos tecnológicos adequados e atualizados.

\section{CONCLUSÃO}

Os profissionais, que participaram da pesquisa, declararam que a SAE traz melhorias para a assistência à medida que ela organiza o trabalho, facilita a visualização dos resultados obtidos a partir do plano de cuidados realizado pelo enfermeiro e confere respaldo e autonomia para o profissional, visto que ele se sente seguro para tomar decisões sobre o tratamento do cliente, já que está embasado em teorias científicas. Entretanto, a sistematização possui barreiras para seu uso como a sobrecarga de trabalho, a resistência por parte dos profissionais de nível técnico e até mesmo dos próprios enfermeiros, por afirmarem não ter tempo para realizá-la e o sistema informatizado, que exige do profissional conhecimento além do seu campo de atuação. Percebeu-se então a necessidade de capacitações e atualizações, para a equipe, abordando aspectos referentes à sistematização, sua importância e forma de aplicá-la, bem como o uso correto da SAE informatizada, para que dessa forma, a equipe de enfermagem possa prestar uma assistência de excelência. Espera-se que este estudo contribua para a reflexão do enfermeiro quanto ao uso da SAE como forma de qualificar a assistência por ele prestada.

\section{REFERÊNCIAS}

1. BARDIN L. Análise de conteúdo. Editora Almedina, Lisboa, Edições 70, LDA, 2009.

2. CASAFUS KCU, et al. Entre o êxito e a frustração com a sistematização da assistência de enfermagem. Esc Anna Nery (impr.), 2013; 17(2):313-321.

3. CHAVES RG, et al. Sistematização da Assistência de Enfermagem: visão geral dos enfermeiros. Rev.Enferm UFPE on line, 2016; 10(4):1280-1285.

4. MARIA MA, et al. Sistematização da assistência de enfermagem em serviços de urgência e emergência: viabilidade de implantação. Rev Bras Enferm, 2012; 65(2): 297-303.

5. MARINELLI NP, et al. Sistematização da Assistência de Enfermagem: desafios para implantação. Revista Enfermagem Contemporânea, 2015; 4(2):254-263.

6. MEDEIROS AL, et al. Sistematização da Assistência de Enfermagem: dificuldades evidenciadas pela teoria fundamentada dos dados. Rev. Enferm. UERJ, 2013; 21(1):47-53.

7. Resolução Cofen-293/2004-Revogada pela Resolução Cofen no 543/207.

8. Resolução Cofen 358 de 2009. Publicado Portal do Cofen - Conselho Federal de Enfermagem.

9. RIBEIRO JC, et al. Informatização da Sistematização da Assistência de Enfermagem: avanços na gestão do cuidado. J. Health Inform, 2014; 6(3): 75-80.

10. SALVADOR PTCO, et al. Ensino da sistematização da assistência de enfermagem aos técnicos de enfermagem. Esc. Anna Nery, 2015; 19(4): 557-562.

11. SANTOS JÁ, et al. Sistematização da Assistência de Enfermagem na visão de enfermeiros. Rev. Cuidarte Enfermagem, 2015; 9(2): 142-147.

12. SENA A, et al. Construção coletiva de um instrumento de cuidados de enfermagem à pacientes no pré operatório imediato. Revista Baiana de Enfermagem, 2017; 31(1):1-10.

13. SILVA CG, et al. Nursing care for patients with chronic health conditions: an integrative review. Rev Fund Care Online, 2017; 9(2): 599-605.

14. SILVA RS, et al. Sistematização da Assistencia de Enfermagem na perspectiva da equipe. Enferm. Foco, 2016; 7 (2): $32-36$.

15. SOARES MI, et al. Sistematização da Assistência de Enfermagem: facilidades e desafios do enfermeiro na gerência da assistência. Esc. Anna Nery, 2015; 19(1): 47-53.

16. TAVARES TS, et al. Avaliação da implantação da sistematização da assistência de enfermagem em uma unidade pediátrica. Rev Min Enferm, 2013; 17(2): 278-286. 